Casos Clínicos

Arch. Esp. Urol. 2010; 63 (10): 871-873

\section{ROTURA DE LA VENA SUPERFICIAL DEL PENE: OPCIONES TERAPÉUTICAS}

Ana Puñal Pereira, Daniel Pérez Fentes, Valentin Toucedo Caamaño, Miguel Blanco Parra, Lorena Villasenín Parrado y Melisa Cortegoso González.

Servicio de Urología. Hospital Universitario de Santiago de Compostela. La Coruña. España.

Resumen.- OBJETIVO: Presentar un caso clínico poco frecuente de rotura de vena dorsal superficial del pene, producida de forma espontánea.

MÉTODO: Paciente de 27 años que acude a urgencias tras la aparición espontánea de gran hematoma y deformidad peneana. La sospecha diagnóstica inicial fue rotura de cuerpos cavernosos.

RESULTADOS: Se realiza exploración quirúrgica, evidenciando la rotura de la vena dorsal superficial del pene, que se liga. Ingreso hospitalario de 24 horas. Completa recuperación estética y funcional a las 2 semanas.
CONCLUSIONES: La rotura de la vena dorsal superficial del pene es una entidad poco frecuente, incluida dentro del diagnóstico diferencial de los hematomas peneanos. La ecografía-doppler peneana puede permitir su diagnóstico y excluir la rotura de cuerpos cavernosos. Aunq-ue el tratamiento conservador parece de elección, ante la duda diagnóstica razonable, está indicada la exploración quirúrgica, la cual ofrece buenos resultados postoperatorios estéticos y funcionales.

Palabras clave: Rotura espontánea. Traumatismos del pene. Diagnóstico. Tratamiento.

Summary.- OBJECTIVE: To report an uncommon clinical case of spontaneous rupture of the superficial dorsal vein of penis.

METHOD: A 27-year-old male patient attended the emergency room following spontaneous occurrence of a large hematoma and deformity in the penis. Rupture of cavernous bodies was initially suspected.

RESULTS: Surgical examination revealed rupture of the superficial dorsal vein of penis, which was ligated. The patient was admitted to hospital for 24 hours, and showed total cosmetic and functional recovery at 2 weeks.

CONCLUSIONS: Rupture of the superficial dorsal vein of penis is an uncommon condition considered in differential diagnosis of penile hematoma. Doppler ultrasound of the penis may allow for its diagnosis and for excluding rupture of corpora cavernosa. Although conservative management appears to be of choice, surgical examination, providing good cosmetic and functional postoperative results, is indicated when a reasonable doubt exists about diagnosis.

Keywords: Spontaneous rupture. Penile physical injuries. Diagnosis. Treatment.

\section{INTRODUCCIÓN}

Las lesiones traumáticas del pene son variadas. La patología más frecuente es la fractura de cuerpos cavernosos. Pero existen patologías vasculares menos frecuentes, que son importantes en el diagnóstico diferencial, ya que pueden imitar su clínica.

\section{CASO CLÍNICO}

Paciente de 27 años intervenido de fimosis como único antecedente de interés, que presenta, de forma espontánea al despertar, un hematoma peneano con leve deformidad del mismo. A lo largo del día presenta progresión de dicho hematoma, por lo que acude al 
servicio de urgencias. El paciente no refiere actividad sexual previa, ni haber escuchado ningún chasquido. Tampoco existe clínica miccional.

En la exploración física existe edema y un hematoma que abarca desde la base del pene hasta la mitad del glande. El pene está claramente deformado, con angulación a la izquierda de la mitad distal. La exploración es dolorosa. Ante esta presentación clínica la hipótesis diagnóstica es rotura de cuerpos cavernosos por lo que el paciente es llevado al quirófano de forma urgente (Figura 1).

Se realiza una incisión subcoronal sobre la cicatriz de postectomía, alcanzando la fascia de Buck y continuando la disección hacia la base. A nivel del tercio medio peneano se encuentra una rotura de la vena dorsal superficial, la cual se liga (Figura 2). Se continúa la exploración quirúrgica, comprobando la integridad de los cuerpos cavernosos.

El paciente fue dado de alta al día siguiente con tratamiento antiinflamatorio y abstinencia sexual. A las 2 semanas se evidencia completa recuperación estética $y$ funcional.

\section{DISCUSIÓN}

Las lesiones vasculares peneanas son poco frecuentes, y ocurren principalmente durante el coito, bien por hiperflexión, bien por traumatismo directo. En menor medida, esto puede suceder al girarse o caer sobre la cama con el pene en erección o durante la masturbación (1). Cuando el pene está en erección, la túnica albugínea pasa de un grosor de $2 \mathrm{~mm}$ a $0.5-0.25 \mathrm{~mm}$ haciéndola mucho más vulnerable a una agresión (2). A la hora de diagnosticar una lesión de este tipo es muy importante realizar una correcta historia clínica y

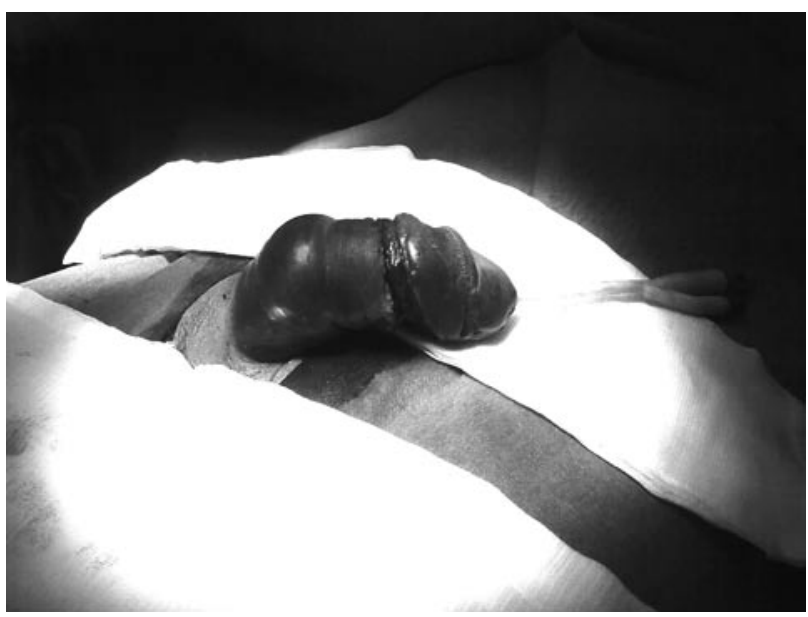

FIGURA 1. Hematoma y deformidad peneana. exploración física. Debemos establecer un diagnóstico diferencial con la fractura de cuerpos cavernosos, la ruptura de la vena dorsal profunda del pene y en algunos casos con la enfermedad de Mondor. Característicamente la fractura de los cuerpos cavernosos presenta un chasquido inicial con dolor inmediato y detumescencia peneana, asociado a equimosis y deformidad hacia el lado no afecto. La palpación es dolorosa y, en ocasiones, puede evidenciarse el lugar de fractura. En casos de afectación uretral el paciente puede presentar uretrorragia y dificultad miccional $(3,4,5)$. La ruptura de la vena dorsal profunda es prácticamente indistinguible de la anterior, salvo que no presenta chasquido inicial y algunas veces cursa sin dolor. A diferencia de ellas la ruptura de la vena dorsal superficial presenta detumescencia gradual y progresivo hematoma generalmente en forma de semiluna, no presenta chasquido, y no suele ser dolorosa ni presentar deformidad peniana $(3,6)$. La enfermedad de Mondor del pene consiste en una flebitis de la vena dorsal superficial, generalmente producida por traumatismos repetidos, $y$ que se manifiesta como un cordón doloroso en la cara dorsal del pene, erecciones dolorosas y equimosis (7).

Ante la duda diagnóstica, conviene realizar una ecografía-doppler peneana, que ofrece información sobre la integridad de la túnica albugínea y permite excluir la presencia de trombosis venosa $(1,6)$. Otras pruebas complementarias, como la resonancia magnéti$\mathrm{ca}$, poco disponible en el contexto de urgencia, o la cavernosografía, ya abandonada por su invasividad, también pueden diagnosticar la rotura de cuerpos cavernosos $(2,5,6)$.

A pesar de que existen pocos casos publicados sobre esta patología, el tratamiento de elección más común es la actitud conservadora con administración de antiinflamatorios, hielo local, cremas heparinoides, vendajes compresivos y antibióticos en algunos casos;

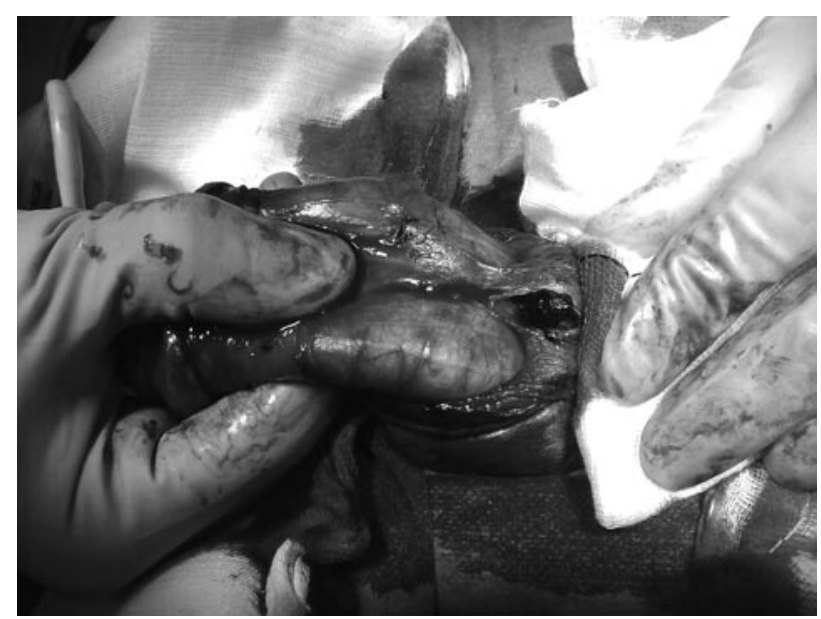

FIGURA 2. Rotura de la vena superficial del pene. 
acompañado de reposo sexual entre 2 y 6 semanas. Se ha visto que con este tratamiento la evolución es buena, con una completa recuperación $(1,2,6)$. No obstante, de existir una duda diagnóstica razonable con la rotura de cuerpos cavernosos, está indicada la exploración quirúrgica urgente $(1,3,8)$.

En nuestro caso, pese a su presentación atípica, el gran hematoma y deformidad peneana nos llevó a dudar sobre la integridad de los cuerpos cavernosos. La revisión quirúrgica permitió excluir su rotura, drenar el hematoma y resolver el sangrado de la vena dorsal superficial. A pesar de optar por una actitud invasiva, se obtuvo una recuperación completa estética y funcional en sólo 2 semanas. Revisando la literatura relacionada, se podría valorar la cirugía en estos pacientes como una opción de tratamiento, ya que la morbilidad es mínima, apenas requiere ingreso hospitalario y la recuperación es muy rápida $(5,8)$.

\section{CONCLUSIONES}

La rotura de la vena dorsal superficial del pene es una entidad poco frecuente, incluida dentro del diagnóstico diferencial de los hematomas peneanos. La ecografía-doppler peneana puede permitir su diagnóstico y excluir la rotura de cuerpos cavernosos. Aunque el tratamiento conservador parece de elección, ante la duda diagnóstica razonable, está indicada la exploración quirúrgica, la cual ofrece buenos resultados postoperatorios estéticos y funcionales.

\section{BIBLIOGRAFÍA Y LECTURAS RECOMENDADAS (*lectura de interés $y^{* *}$ lectura fundamental)}

*1. Arce Gil J, Rodríguez- Ledesma JM, Villavicencio Mavrich H. Rotura de la vena dorsal superficial de pene. Actas Urol Esp, 2006; 30(2): 215-217.

$* * 2$. Sharma GR. Rupture of the superficial dorsal vein of the penis. Int J Urol, 2005; 12(12): 1071-1073.

**3. Bar-Yosef Y, Greenstein A, Beri A, Lidawi G, Matzkin H, Che-n J. Dorsal vein injuries observed during penile exploration for suspected penile fracture. $\mathrm{J}$ Sex Med, 2006; 4 (4Pt2): 1142-6.

4. Martínez RJ, Pastor Navarro H, Carrión López P, Giménez Bachs JM, Donate MM, Virseda Rodríguez JA. Fractura de cuerpos cavernosos. Serie de casos. Actas Urol Esp, 2008; 32(6): 599-602.

5. Shah DK, Paul EM, Meyersfield SA, Schoor RA. False fracture of the penis". Urol, 2003; 61(6):1259.

*6. Bujons Tur A, Rodríguez-Ledesma JM, Cetina Errando A, Puigvert Martínez A, Iglesias Guzman JC, Villavicencio Mavrich H. Hematoma peneano secundario a rotura de la vena dorsal superficial del pene. Arch Esp Urol, 2004; 57 (7): 748-751.
7. Molina Escudero R, Cabello Benavente R, Monzó Gardiner JI, López Díez I, Tabares Jimenez J, Paños Fagundo E, Hernández Fernández C. Mondor's syndrome. Case review and bibliographic re-view. Arch Esp Urol, 2009; 62 (4): 317-9.

8. Feki W, Derouiche A, Belhaj K, et al. False penile fracture: report of 16 cases. Int J Impot Res, 2007; 19(5):471-3. 